INSTITUT NATIONAL DE RECHERCHE EN INFORMATIQUE ET EN AUTOMATIQUE

\title{
Geometry of information propagation in massively dense ad hoc networks
}

Philippe Jacquet

$\mathbf{N}^{\circ} 4992$

THÈME 1 



\title{
Geometry of information propagation in massively dense ad hoc networks
}

\author{
Philippe Jacquet * \\ Thème 1 - Réseaux et systèmes \\ Projet Hipercom \\ Rapport de recherche $n^{\circ} 4992--10$ pages
}

\begin{abstract}
Using the fact that effective wireless range decraise in inverse function of local traffic density, we show that a variable traffic density impacts the curvature of paths in a dense wireless ad hoc network the same way a variable optical density bends light paths. We set up the general laws that paths must satisfy in presence of traffic flow density. We quantify the effect and we give some example of configurations treated analytically.
\end{abstract}

Key-words: Mobile ad hoc, packet propagation, optimal routes, path curvatures.

\footnotetext{
* (philippe.jacquet@inria.fr)
} 


\section{Géométrie de la propagation de l'information dans les réseaux mobiles ad hoc massivement denses}

Résumé : Nous utilisons le fait que la portée effective dans les réseaux sans fil décroît en raison inverse de la densité locale du trafic, nous montrons que toute variation spatiale de la densité de trafic joue sur la courbure des trajets de propagation des paquets dans des réseaux mobiles ad hoc denses, un peu comme un indice optique variable courbe les faisceaux lumineux. Nous quantifions l'effet et nous traitons analytiquement quelques exemples simples.

Mots-clés : Mobile ad hoc, propagation de paquets, routes optimales, courbures de trajets. 


\section{Introduction}

In their reference paper on the capacity of mobile ad hoc networks, Gupta and Kumar [3] showed that the radius of correct reception decays in inverse of square root of radio emitter density. This estimate was originally derived from information theory considerations and is not related to any particular network implemetation. In [1] we rederived this results in a particular network implementation where time is slotted and transmitter are dispatched randomly and uniformly on a planar map. Let $\lambda$ be the density of transmitter per unit area and per slot, $\lambda$ is expressed in bit per square meter per slot. It turns out that the radius of the correct reception of a packet within a certain probability is equal to $\frac{\beta}{\sqrt{\lambda}}$ where quantity $\beta$ depends on the given probability, the attenuation coefficient of wave propagation and the signal-over-noise ratio required for correct reception (see [1] for more details). This comes from the fact that the disk of correct reception within a given probability contains a finite number of transmitters, thus has an area proportional to $\frac{1}{\lambda}$.

If we consider a network dispatched a map of dimension $D$ then the estimate of the radius will be $\frac{\beta}{\lambda^{1 / D}}$. In the sequel we will look at $2 \mathrm{D}$ maps generalizing occasionnaly the results on any dimensional maps. When the density $\lambda$ increases in a map kept constant then, the minimum number of hops connecting two points $A, B$ tends to be equivalent to $d(A, B) \frac{\sqrt{\lambda}}{\beta}$ where $d(A, B)$ denotes the euclidian distance between mobile $A$ and mobile $B$. Meanwhile, the increase of the number of relays naturally increases the traffic density. If $\nu$ is the actual traffic density generation per unit area, i.e. the traffic locally generated on mobile nodes, not the traffic relayed by the mobile nodes, then the average density traffic will satisfy the identity: $\lambda=\nu \bar{d} \frac{\sqrt{\lambda}}{\beta}$ where $\bar{d}$ is the average euclidian distance between two end points in a connection.

This previous identity assumes that the pattern of path between points covers the map in an uniform manner so that the traffic density, generated and relayed, is constant on the whole map. In this case the path that connect two points with the minimum number of hops is very close to the straight line. But the question arises about the shape of the optimal path when the traffic density is not uniform. This paper will address the case where the traffic pattern is not uniform and when the traffic density is high enough that it makes the efficient radio ranges infinitely small (in the scale of the map). We will address the problem on its macroscopic aspect where connection paths only affect traffic density at infinitely small scale. In other words we deal here with asymptotic case.

no mobility, so far, etc. Problem with optimal path affecting density, and vice versa.

\section{Massively dense networks}

We consider massively dense networks on a 2D map. We denote by $\lambda(x, y)$ the traffic density at point of coordinate $(x, y)$ on the map. We suppose that function $\lambda(x, y)$ is continuous in $(x, y)$, or at least Lebesgue integrable. When $\lambda(x, y)$ are uniformly large then the radio ranges tend to be "microscopic" and routes can be considered as continuous lines between nodes. Packets travelling on a route $\mathcal{C}$ passing over point of coordinate $(x, y)$ will experience hops of

$\mathrm{RR} \mathrm{n}^{\circ} 4992$ 
length $\frac{\beta}{\sqrt{\lambda(x, y)}}$ when passing in the proximity of point $(x, y)$. Let $n(x, y)=\frac{\sqrt{\lambda}}{\beta}$, the number of hops that a packet must experience on route $\mathcal{C}$ is close to the integral: $\int_{\mathcal{C}} n(x(s), y(s)) d s$ where $s$ is a curvilign absciss on route $\mathcal{C}$.

In the sequel we are looking for route with the shortest hop number and we conjecture that it is optimal in terms of traffic balancing and local performance in terms of delay and throughput. Of course this comes with the assumption that any new connections provides only infinitesimal increment of the traffic density. Searching the path that minimize the hop number between two points $A$ and $B$ is therefore equivalent for looking for the path light between $A$ and $B$ in a medium with non-uniform optical index $\lambda(x, y)$. It is known that the optimal path satisfies on each of its point $\mathbf{z}(s)=(x(s), y(s))$ :

$$
\frac{d}{d s}\left(n(\mathbf{z}(s)) \frac{d \mathbf{z}(s)}{d s}\right)=\nabla n(\mathbf{z}(s))
$$

where $\nabla$ is symbol of gradient vector. Therefore finding the optimal path is just an application of geometric optics.

However we face a major problem in the fact that the distribution of path is actually impacting traffic density. This lead to an egg-and-chicken problem which may not that easy to solve. We call $\Phi(x, y)$ the flow density of information transiting in the vicinity of point $(x, y)$. Quantity $\Phi(x, y)$ is expressed in bit per meter, since it express the flow of packet crossing a virtual unit of segment of length of 1 meter centered on point $(x, y)$. This flow impact the traffic density by the fact that each packet must be relayed each $\beta / \sqrt{\lambda(x, y)}$ in the vicinity of point $(x, y)$. Therefore locally:

$$
\lambda(x, y)=\Phi(x, y) \frac{\sqrt{\lambda(x, y)}}{\beta}
$$

In other words $\lambda(x, y)=\left(\frac{\Phi(x, y)}{\beta}\right)^{2}$ and

$$
n(x, y)=\frac{\Phi(x, y)}{\beta^{2}}
$$

When considering map of dimension $D$ we have $\lambda=\Phi \frac{\lambda^{1 / D}}{\beta}$ and $n=\left(\frac{\Phi}{\beta^{D}}\right)^{\frac{1}{D-1}}$. Notice that the equations are singular when $D=1$.

As an example we can assume a planar map massively and uniformly filled with mobile nodes and gateway nodes. We denote by $\mu_{G}$ the spatial density of gateways. We assume that the mobile nodes are much more dense than the gateways. We denote by $\nu$ the traffic density generated in any point. $\nu$ is expressed in bits per square meters per slot. The flow density $\Phi$ is constant in the map and is equal to $\nu \bar{d}$. We suppose that mobile nodes sends and receives flows from their closest gateway. Therefore $\bar{d}=\int_{0}^{\infty} \exp \left(-\pi \mu_{G} r^{2}\right) d r=\frac{1}{2 \sqrt{\mu_{G}}}$ :

$$
\Phi=\frac{\nu}{2 \sqrt{\mu_{G}}}
$$




\section{Examples}

As a general framework we rewrite equation (1) that we rewrite in

$$
n \frac{d}{d s}\left(n \frac{d \mathbf{z}}{d s}\right)=\frac{\nabla n^{2}}{2}
$$

This equation has a straightforward analogy with dynamics. It turns out that if a particle moves at speed $n(\mathbf{z})$ on every point $(\mathbf{z})$, then traveling on an optimal path implies that the particle is submitted to an acceleration $\frac{\nabla n^{2}(\mathbf{z})}{2}$, or, in other words, it is submitted to a potential $\frac{n^{2}(\mathbf{z})}{2}$.

\subsection{The central gateway}

We suppose that the map is a disk of radius $R$ where nodes on the border maintain permanent and identical flows to a gateway located in the center of the disk. Our aim is to describe the shortest path between two points $A$ and $B$ which are inside the disk.

Nodes on the outer circle transmit datas toward the central gateway (or equivalently receive data from the central gateway). By symmetry considerations, the shortest path between the gateway and any node on the circle just follows the radius drawn from the disk center to this node. Thus we have the identity:

$$
\Phi(\mathbf{z})=\ell \frac{R}{|\mathbf{z}|}
$$

where $\ell$ is the linear density of traffic generated on the circle, expressed in bit per meter per slot. We notice that there is a singularity when $\mathbf{z}=0$. We can cope with this singularity by removing from the map a disk of smaller radius $r<R$, and imagining that the inner circle contains gateways uniformly distributed. Nodes access their closest gateway and their path follow the radius.

If we take the analogy with dynamics, the optimal path between two points is the trajectory of a moving body at speed $|\dot{\mathbf{z}}|=n(\mathbf{z})=\frac{\ell R}{\beta^{2} \mathbf{z} \mid}$ and acceleration $\ddot{\mathbf{z}}=\frac{1}{2} \nabla n^{2}(\mathbf{z})=$ $-\left(\frac{\ell}{\beta^{2}|\mathbf{z}|^{2}}\right)^{2} \mathbf{z}$. Since the aceleration is central, i.e. is colinear with the vector $\mathbf{z}$, Kepler's law of area conservation applies: The area of the triangle made by vector $\mathbf{z}$ and vector $\dot{\mathbf{z}}$ is constant on the body trajectory. Since this area is equal to $\frac{\ell \sin \theta}{\beta^{2}}$ where $\theta$ is the angle between radius vector and speed vector. It turns out from Kepler's law that $\theta$ is constant and we can state the theorem:

Theorem 1 The optimal path between two points $A$ and $B$ in the central gateway configuration is the portion of logarithmic spiral centered on the gateway that connect the two points.

Corollary 1 When the two points are at same distance to the gateway, then the optimal path is the portion of circle centered on the gateway connecting the two points.

$\mathrm{RR} \mathrm{n}^{\circ} 4992$ 
The consequence of this is that the optimal path is never the straight line and when the point are opposite with respect to the central gateway, then the optimal path starts orthogonally to the linear segment connecting the points.

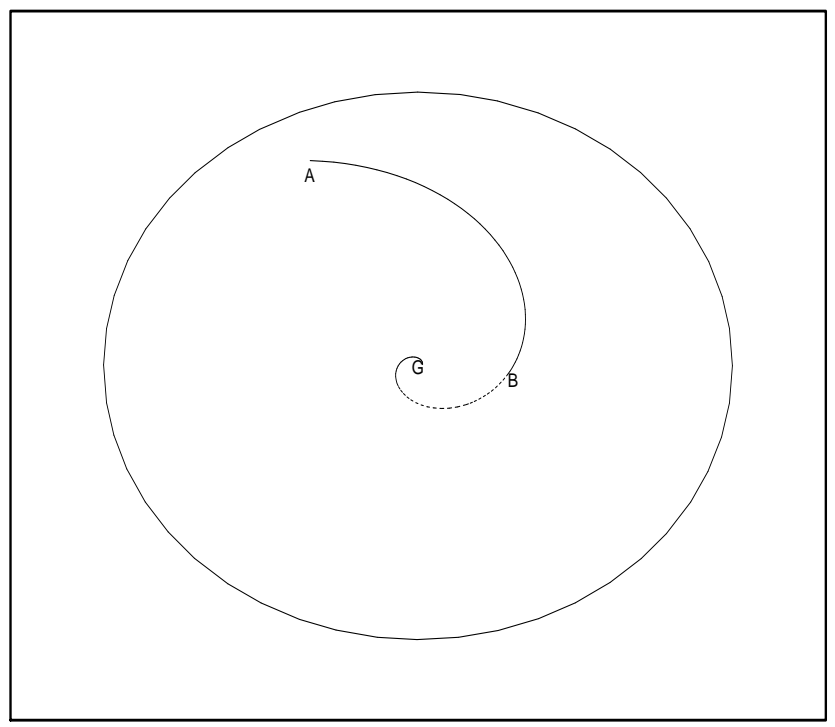

Figure 1: Optimal path between points $A$ and $B$ in the central gateway configuration.

\subsection{The border gateways}

We take the same disk map as in the previous example. Now we assume that the gateways are densely and uniformly distributed on the border of the outer circle and all nodes generates uniform traffic toward their closest gateway, i.e the gateway which is on the radius of the circle that contains the node. Due to symmetry consideration, the shortest path to the gateway is the radius segment. Thus easy algebra gives

$$
\Phi(\mathbf{z})=\ell \frac{|\mathbf{z}|}{R}
$$

INRIA 


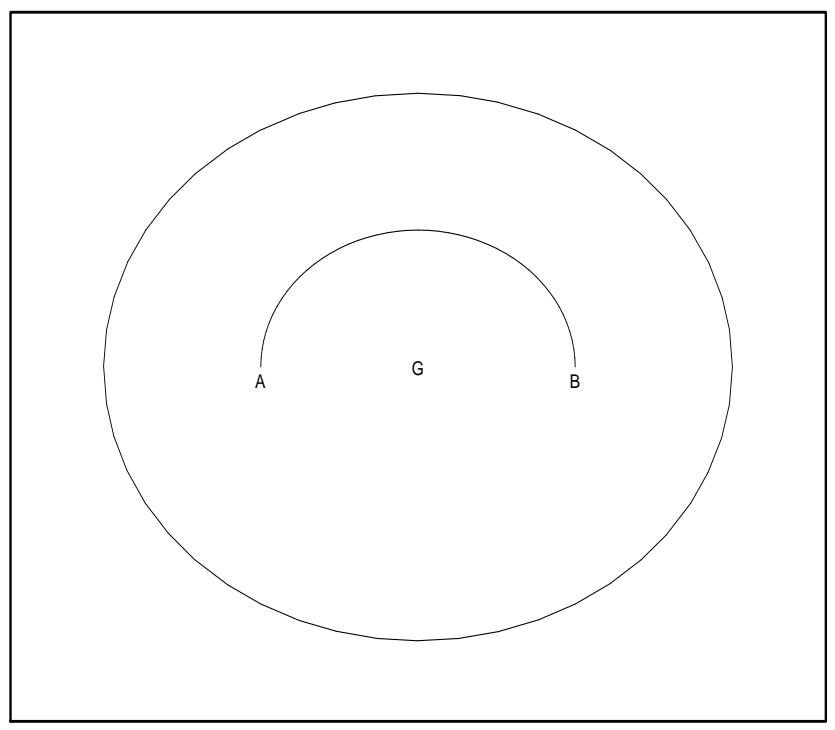

Figure 2: Optimal path between two opposite points $A$ and $B$ in the central gateway configuration.

where $\ell$ is the linear density of information arriving on the outer circle. Taking the analogy with the body in motion, an optimal path is the trajectory of the body in motion such that $|\dot{\mathbf{z}}|=\gamma|\mathbf{z}|$ and $\ddot{\mathbf{z}}=\gamma^{2} \mathbf{z}$ with $\gamma=\frac{\ell}{R \beta^{2}}$.

The solution of the differential equation on time variable $t$ is the form $\mathbf{z}=e^{\gamma t} \mathbf{u}+e^{-\gamma t} \mathbf{v}$ where $\mathbf{u}$ and $\mathbf{v}$ are arbitrary vectors. The identity $|\dot{\mathbf{z}}|=\gamma|\mathbf{z}|$ implies that the vectors $\mathbf{u}$ and $\mathbf{v}$ must be orthogonal. Consequently an optimal path must be a branch of hyperbol. However a branch of hiperbol can only connect nodes $A$ and $B$ that form an angle smaller than $\frac{\pi}{2}$ with the disk center. When the angle is larger then the shortest path is simply made of the union of the two segments connecting the center with the nodes $A$ and $B$.

Theorem 2 In the border gateway configuration on a disk of center $O$, the optimal path between two nodes $A$ and $B$ is the following:

(i) When the angle in $O$ of the triangle $(A, B, O)$ is less than $\frac{\pi}{2}$, then the optimal path is the branch of hiperbol that connect the two points $A$ and $B$;

$\mathrm{RR} \mathrm{n}^{\circ} 4992$ 
(ii) When the angle in $O$ of the triangle $(A, B, O)$ is larger than or equal to $\frac{\pi}{2}$, then the optimal path is the union of the segments $(A, O)$ and $(O, B)$.

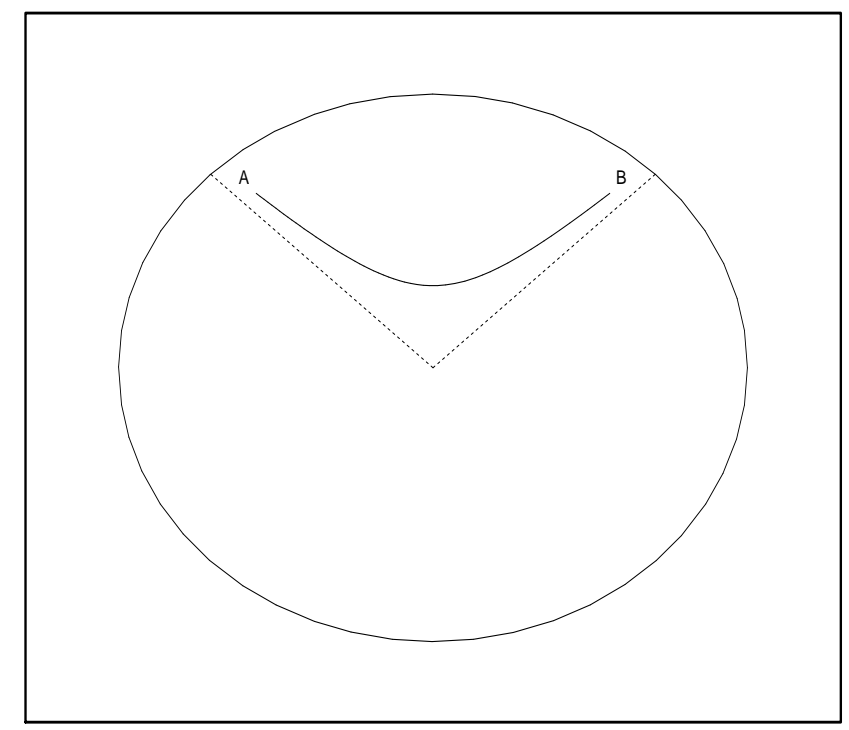

Figure 3: Optimal path between two points $A$ and $B$ with angle with center smaller than $\frac{\pi}{2}$, in the outer gateways configuration.

\section{Information flow tensor}

The previous examples were tractable because the path to the gateways were determined $a$ priori by symmetry considerations and are not disturbed by the variation of parameter $n(\mathbf{z})$. In fact the problem is that the information path and the traffic density mutually affect each other. We have to describe the information flow in more details. Let consider a point $(x, y)$, let $\mathbf{u}_{i}$ be the sequence of information flow vectors passing in the vicinity of this point, each vector is expressed in bit per meter. We define the tensor of information flow vector $\mathbf{T}$ the 


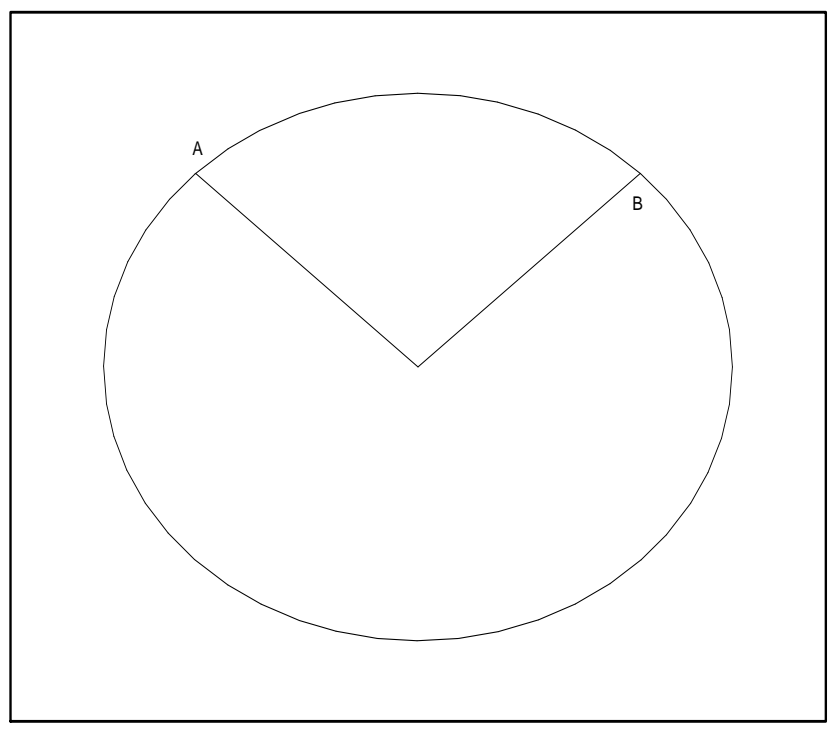

Figure 4: Optimal path between two points $A$ and $B$ with angle with center equal to $\frac{\pi}{2}$, in the outer gateways configuration.

following:

$$
\mathbf{T}(x, y)=\sum_{i} \frac{1}{\left|\mathbf{u}_{i}\right|} \mathbf{u}_{i} \otimes \mathbf{u}_{i}
$$

The definition is equivalent to the tensor stress-energy in physics. We have $\Phi=\operatorname{tr}(\mathbf{T})=$ $\sum_{i}\left|\mathbf{u}_{i}\right|$. The divergence of $\mathbf{T}$ can be interpreted as local generation of information flow: $\nabla \cdot \mathbf{T}=\mathbf{v}$ where $\mathbf{v}$ is the sum of all information vectors generated in the vivinity of point $(x, y)$. An information vector $\mathbf{v}$ is when information is generated in volume $|\mathbf{v}|$ on point $(x, y)$ and is transmitted toward a path with initial vector $\frac{\mathbf{v}}{\mid \mathbf{v}}$.

\section{References}

[1] C. Adjih, E. Baccelli, P. Jacquet, "Link State Routing in Wireless Ad-Hoc Networks," milcom 2003, Boston.

$\mathrm{RR} \mathrm{n}^{\circ} 4992$ 


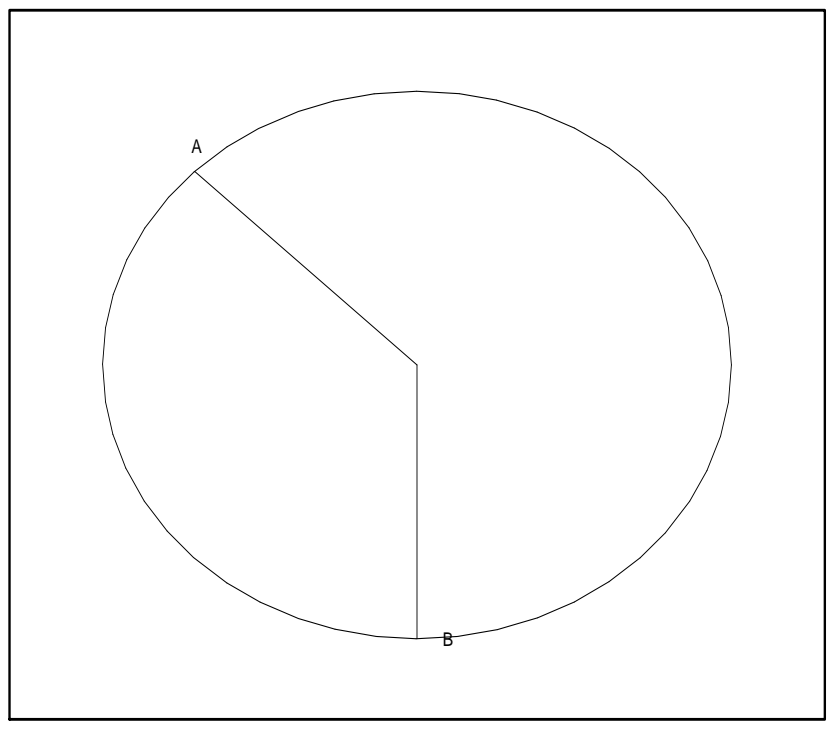

Figure 5: Optimal path between two points $A$ and $B$ with angle with center greater than $\frac{\pi}{2}$, in the outer gateways configuration.

[2] P. Jacquet, "Elément de théorie analytique de l'information, modélisation et évaluation de performances," INRIA Research Report RR-3505, http://www.inria.fr/rrrt/rr3505.html, 1998.

[3] P. Gupta and P.R. Kumar. Capacity of wireless networks. Technical report, University of Illinois, Urbana-Champaign, 1999. http://citeseer.nj.nec.com/gupta99capacity.html 


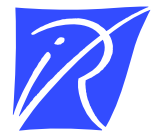

Unité de recherche INRIA Rocquencourt Domaine de Voluceau - Rocquencourt - BP 105 - 78153 Le Chesnay Cedex (France)

Unité de recherche INRIA Lorraine : LORIA, Technopôle de Nancy-Brabois - Campus scientifique 615, rue du Jardin Botanique - BP 101 - 54602 Villers-lès-Nancy Cedex (France)

Unité de recherche INRIA Rennes : IRISA, Campus universitaire de Beaulieu - 35042 Rennes Cedex (France)

Unité de recherche INRIA Rhône-Alpes : 655, avenue de l'Europe - 38330 Montbonnot-St-Martin (France)

Unité de recherche INRIA Sophia Antipolis : 2004, route des Lucioles - BP 93 - 06902 Sophia Antipolis Cedex (France)

INRIA - Domaine de Voluceau - Rocquencourt, BP 105 - 78153 Le Chesnay Cedex (France)

http://www.inria.fr

ISSN 0249-6399 